\title{
Dimorphisme sexuel et cavités faciales: étude volumétrique en imagerie 3D
}

\author{
Nayla BassiL-NassiF ${ }^{1 *}$, Joseph Bouserhal ${ }^{1}$, Jacques TreiL ${ }^{2}$, José Braga ${ }^{3}$, Robert Garcia ${ }^{4}$ \\ 1 Département d'Orthodontie, Faculté de Médecine Dentaire, Université Saint-Joseph, Rue de Damas, Beyrouth, Liban \\ 2 Faculté de Médecine Dentaire, Université de Toulouse III, 3 rue des Maraîchers, 31400 Toulouse Cedex 9, France \\ ${ }^{3}$ Laboratoire d'Anthropobiologie, Université Toulouse III, 37 Allées Jules Guesde, 31000 Toulouse, France \\ ${ }^{4}$ Faculté de Médecine Dentaire, Paris VII, 5 rue Garancière, 75006 Paris, France
}

(Reçu le 14 janvier 2010, accepté le 11 mars 2010)

MOTS CLÉS :

Dimorphisme sexuel /

Scanner 3D /

Volumes des cavités

cranio-faciales

KEYWORDS:

Sexual dimorphism /

CT Scan /

Craniofacial cavity volume
RÉSUMÉ - Introduction : L'objectif de ce travail est d'étudier le dimorphisme sexuel au niveau des volumes des cavités faciales dans une population adulte jeune. Méthodes : L'échantillon sélectionné comporte 60 sujets, âgés entre 18 et 30 ans, ayant bénéficié d'un examen scanner de la tête. Ils sont divisés en deux groupes identiques selon le sexe. Les cavités orbitaires, ethmoïdo-nasale et buccale sont délimitées par des points sur les acquisitions scanner 3D et leurs volumes mesurés à l'aide du logiciel AMIRA ${ }^{\circledR}$, alors que les deux sinus maxillaires sont segmentés et leurs volumes calculés. Le test Kolmogorov-Smirnov est utilisé pour démontrer la normalité des variables, suivi du test « $t$ » de Student pour la comparaison des moyennes $(p<0,05)$. Résultats : Les moyennes des volumes des cavités orbitaires, ethmoïdo-nasales, buccales et des sinus maxillaires sont plus grandes chez les hommes, alors que le dimorphisme sexuel n'a pas été mis en évidence au niveau du rapport des volumes de chacune des cavités sur le volume facial total. Conclusion : Les volumes absolus des cavités faciales étudiées sont plus grands chez les hommes, mais pas les volumes relatifs, ce qui pourrait s'expliquer par l'existence d'une différence craniofaciale de taille, et non de forme, entre les hommes et les femmes.

ABSTRACT - Sexual dimorphism and facial cavities: A 3D imaging volumetric study. Introduction: The aim of this study was to investigate the sexual dimorphism in facial cavity's volumes of untreated young adults. Methods: Sixty subjects, ages between 18 to 30 years, were selected and divided in two groups according to the gender. Volumes of orbital, nasal, sinusal and buccal cavities were measured from CT scans with AMIRA ${ }^{\circledR}$ software. The statistical studies were performed using Kolmogorov-Smirnov test for normality followed by Student " $t$ " test $(p<0.05)$. Results: The gender comparison reflected statistically significant differences between the volumes of orbital, nasal, sinusal and buccal cavities, volumes being larger in males. Sexual dimorphism did not appear in the ratio of each cavity volume on the total facial volume. Conclusion: Absolute volume of facial cavities are larger in males, but no sexual dimorphism is detected comparing volume ratios. A difference in size, but not in shape, between genders might exist.

\section{Introduction}

La morphologie et la croissance cranio-faciales ont été longuement étudiées depuis l'avènement des techniques céphalométriques. De nombreuses

\footnotetext{
* Auteur pour correspondance : nbassilnassif@yahoo.com
}

études ont été faites pour comprendre l'évolution des structures et leurs directions de croissance, mais cela sur des radiographies en 2D qui ne peuvent mesurer que deux dimensions du complexe craniofacial de forme volumétrique, alors que la troisième 
dimension reste toujours manquante. Houston [19], dans son étude sur l'analyse des erreurs en céphalométrie, a soulevé le problème des inexactitudes inhérentes aux radiographies, à leur interprétation et aux mesures céphalométriques, qu'elles soient dues à l'identification des structures ou à leur superposition. Le problème devient plus important, comme l'ont souligné Kau [21] et Sosa [29], et les résultats moins fiables, lorsqu'il s'agit d'étudier des cavités cranio-faciales. En orthopédie dento-faciale, des constructions céphalométriques ont été mises au point dont celles de Treil, et al. [31] et Faure, et al. [7-10] à partir d'acquisitions tomodensitométriques issues d'un appareil scanner, ou Cho, et al. [2] à partir d'images issues d'un appareil cone-beam, en utilisant différents logiciels informatiques. Aussi, plusieurs études ont été menées en 3D dans différents domaines, par Hennessy et Moss [18], et Kau et Richmond [21], sur la croissance cranio-faciale, Nabbout, et al. [24] sur les volumes des dents, Sampeur et Boileau [25] sur les muscles masticateurs ainsi que Dah-Jouonzo, et al. [3,4] sur les mesures volumétriques des bases osseuses et des cavités aériennes de la face. D’autres auteurs, Kau, et al. [22,23], Dean, et al. [5], Ferrario, et al. [11-13], ainsi que Sforza, et al. [26], ont essayé d'étudier les structures osseuses, les tissus mous ou même la morphologie faciale en s'aidant d'outils capables de fournir des résultats en 3D.

Mais l'aspect volumétrique du dimorphisme sexuel n'a pas encore été abordé, bien que les études en 2D aient trouvé une différence en taille, direction de croissance et pic de maturation entre les deux sexes $[1,15,33]$. Dans leur étude sur la morphologie crânienne à partir de mesures faites sur des radiographies, Ingerslev et Solow [20] ont montré que le crâne des hommes était en moyenne plus grand, mais que les femmes présentaient un front plus saillant et un os nasal moins proéminent. Ursi, et al. [32], en étudiant le dimorphisme sexuel pendant la croissance sur des téléradiographies de profil prises entre 6 et 18 ans à des intervalles de trois ans, ont conclu que la partie antérieure de la base du crâne est plus grande chez les hommes sans qu'il n'y ait une différence au niveau de l'angle basal entre les deux sexes; les positions sagittales du maxillaire et de la mandibule sont identiques, ainsi que leur croissance en longueur jusqu'à l'âge de 14 ans, âge à partir duquel cette longueur reste relativement constante chez le sexe féminin, alors qu'elle augmente chez les hommes; les directions de croissance sont identiques au niveau des deux groupes, avec une tendance vers une direction plus horizontale chez les femmes.

Le but de notre étude est de comparer, en 3D, les volumes des cavités faciales: orbitaires, ethmoïdonasale, buccale ainsi que les sinus maxillaires, entre deux groupes de sujets adultes jeunes de sexe différent, à partir d'acquisitions scanner et à l'aide du logiciel AMIRA ${ }^{\circledR}$.

\section{Matériel et méthodes}

L'échantillon d'étude comporte 60 sujets ayant bénéficié d'un scanner de la tête. Pour être sélectionnés, ils devaient avoir entre 18 et 30 ans d'âge, une denture permanente complète et ne pas avoir bénéficié de traitement orthodontique préalable. Les personnes qui présentaient une pathologie sinusienne, des séquelles de traumatisme osseux facial ou un syndrome causant une déformation squelettique n'ont pas été inclus. Deux groupes formés de 30 sujets chacun, de sexe différent, ont pu être constitués.

Les examens scanners, qui sont traités dans le cadre de l'étude, avaient été effectués au service d'Imagerie Médicale AMI, avec le même appareil Helical CT scanner « Philips Tomoscan AV », l'épaisseur des coupes étant de $1 \mathrm{~mm}$. Les données numériques des coupes natives sont enregistrées en format DICOM (Digital Imaging for COmmunication in Medicine).

Avec le logiciel AMIRA ${ }^{\circledR}$, utilisé dans notre étude, nous avons pu obtenir une reconstruction tridimensionnelle de chaque sujet, à partir des coupes natives. Ceci nous a permis d'identifier et de saisir les points que nous avons choisis pour délimiter les cavités faciales à étudier : cavités orbitaires, ethmoïdonasale et buccale (Fig. 1 et 2):

- Les foramens supra- et infra-orbitaires (FSOD, FSOG, FIOD, FIOG), le canthus externe (CED, CEG), l'orifice supérieur du canal lacrymo-nasal (CLND, CLNG) et l'orifice du canal optique (COD, COG) délimitent les cavités orbitaires respectivement droite et gauche (A et B).

- Les orifices des canaux optiques et les parties supérieures des foramens infra-orbitaires droits et gauches constituent le plafond de la cavité 


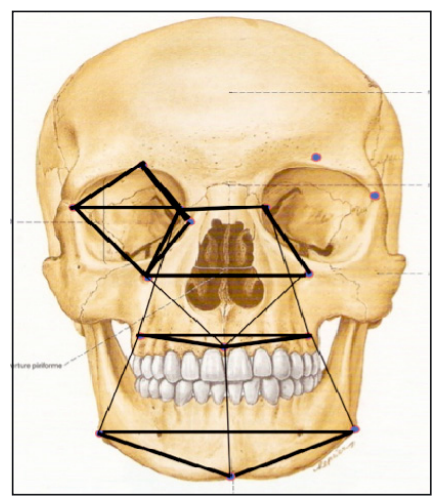

a

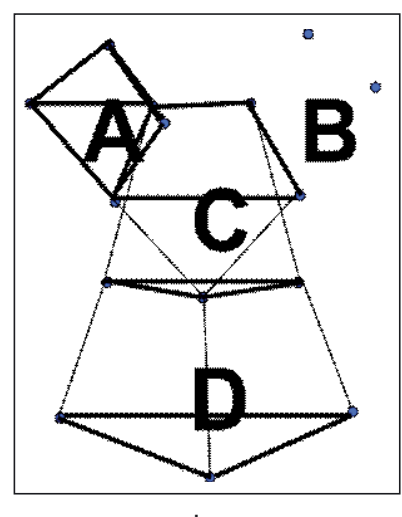

b

Figure 1

(a) Localisation des points et schématisation des volumes des cavités orbitaire, ethmoïdo-nasale et buccale : vue de face (squelette crânien d'après Sobotta [28]). (b) Modèle géométrique délimitant les cavités orbitaire, ethmoïdo-nasale et buccale : vue de face.

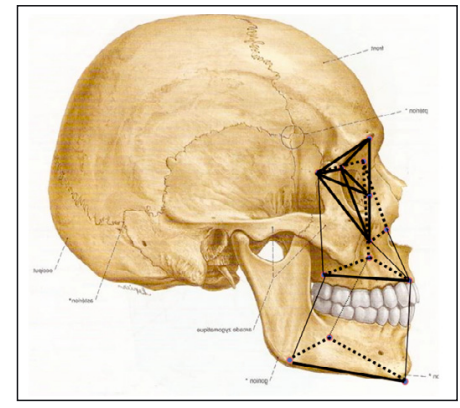

a

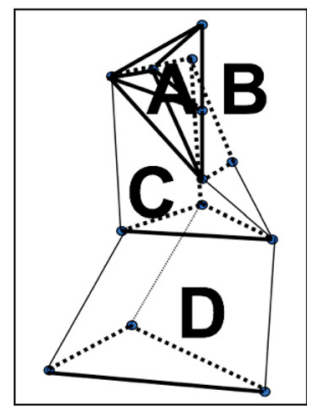

b

Figure 2

(a) Localisation des points et schématisation des volumes des cavités orbitaire, ethmoïdo-nasale et buccale : vue de profil (squelette crânien d'après Sobotta [28]). (b) Modèle géométrique délimitant les cavités orbitaire, ethmoïdo-nasale et buccale : vue de profil.

ethmoïdo-nasale (C), alors que son plancher est formé par les parties antéro-médianes des foramens grands palatins droit et gauche (FGPD, FGPG) et du foramen naso-palatin (FNP).

- Le plafond de la cavité buccale (D) est formé par le plancher de la cavité ethmoïdo-nasale, décrit précédemment, et son plancher par les parties les plus inféro-postérieures, en vue latérale, des gonions droit et gauche (GD, GG), et de la partie la plus inféro-antérieure de la pointe symphysaire (PS).

Ces points de repère localisés, leurs coordonnées $X, Y$ et $Z$ ont été directement identifiées, et le volume construit délimité par ces points et préfigurant la cavité étudiée, a été trouvé à l'aide d'un logiciel de calcul mis au point à cet effet, l'unité volumétrique étant le $\mathrm{cm}^{3}$.
Les sinus maxillaires étant des cavités bien délimitées, c'est la sélection manuelle qui a été choisie, bien que laborieuse et consommatrice de temps, mais plus précise pour les structures «fermées ». La segmentation s'est faite coupe par coupe, par remplissage de la cavité, à l'aide d'un outil choisi en fonction de la taille et la forme nécessaires. Le volume total des sélections faites sur les coupes, qui constitue le volume du sinus étudié, est donné directement par le logiciel. Il est à noter que, pour les cavités paires, c'est le volume total constitué de la somme des deux cavités droite et gauche qui est étudié (Fig. 3). La somme des volumes des deux cavités orbitaires, des cavités ethmoïdo-nasale et buccale, et des deux sinus maxillaires forme le volume global des cavités faciales.

Un passage des coupes natives à la reconstruction tridimensionnelle peut se faire très facilement, 
Tableau 1

Statistiques descriptives et comparaison des volumes entre les deux groupes hommes/femmes.

\begin{tabular}{|c|c|c|c|c|c|c|c|}
\hline & Groupe & $N$ & Minimum & Maximum & Moyenne & Ecart-type & $p$ \\
\hline \multirow{2}{*}{$\begin{array}{l}\text { Volume cavités } \\
\text { orbitaires }\end{array}$} & Homme & 30 & 8,056 & 29,265 & 19,99047 & 5,067658 & \multirow{2}{*}{0,022} \\
\hline & Femme & 30 & 5,644 & 23,561 & 17,03107 & 4,672435 & \\
\hline \multirow{2}{*}{$\begin{array}{l}\text { Volume cavité } \\
\text { ethmoïdo-nasale }\end{array}$} & Homme & 30 & 28,000 & 51,247 & 37,78417 & 5,636993 & \multirow{2}{*}{0,000} \\
\hline & Femme & 30 & 22,842 & 43,976 & 30,36200 & 4,160728 & \\
\hline \multirow{2}{*}{$\begin{array}{l}\text { Volume cavité } \\
\text { buccale }\end{array}$} & Homme & 30 & 54,399 & 129,641 & 95,62673 & 12,666033 & \multirow{2}{*}{0,000} \\
\hline & Femme & 30 & 59,209 & 89,969 & 74,07207 & 7,609101 & \\
\hline \multirow{2}{*}{$\begin{array}{l}\text { Volume sinus } \\
\text { maxillaires }\end{array}$} & Homme & 30 & 13,494 & 62,231 & 40,89882 & 9,530240 & \multirow{2}{*}{0,000} \\
\hline & Femme & 30 & 18,425 & 48,694 & 32,21326 & 7,926346 & \\
\hline \multirow{2}{*}{$\begin{array}{l}\text { Volume } \\
\text { total }\end{array}$} & Homme & 30 & 161,944 & 239,712 & 194,30118 & 18,609302 & \multirow{2}{*}{0,000} \\
\hline & Femme & 30 & 123,483 & 189,620 & 153,68130 & 17,171397 & \\
\hline
\end{tabular}

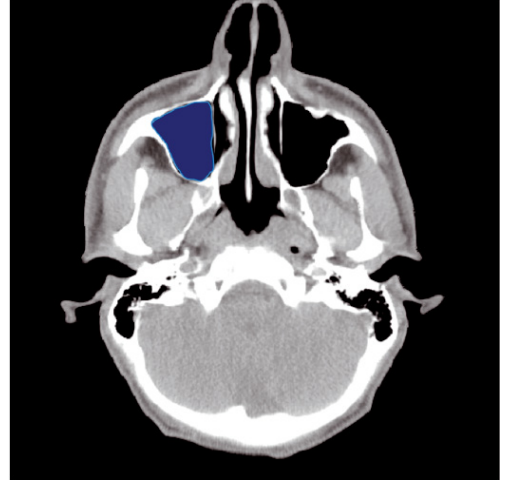

Figure 3

Segmentation du sinus droit au niveau d'une coupe selon l'orientation $x y$.

pendant le travail, ce qui a permis de contrôler l'exactitude du point choisi pour les cavités construites ou de la sélection manuelle pour les sinus maxillaires.

L'erreur intra-opérateur, calculée à l'aide de l'Intraclass Correlation Coefficient (ICC) sur vingt examens scanners choisis au hasard, a montré des valeurs totalement négligeables.

L'étude statistique est effectuée d'un point de vue descriptif à partir des données recueillies sur les 60 acquisitions scanners analysées. Les quatre variables, que sont les volumes des cavités orbitaires, des sinus maxillaires, de la cavité ethmoïdo-nasale et de la cavité buccale, sont comparées entre les deux groupes avec un risque d'erreur $\alpha$ de $5 \%$ et une puissance de $80 \%$. Les tests ont été réalisés avec le logiciel de statistiques SPSS ${ }^{\circledR}$ version 15 : le test Kolmogorov-Smirnov pour démontrer la normalité des variables, suivi du test $« t »$ de Student pour la comparaison des différentes moyennes. Chaque test a été considéré significatif lorsque $p<0,05$.

\section{Résultats}

Les comparaisons des valeurs moyennes et des écarts-types des différentes cavités et du volume global entre les deux groupes hommes et femmes ont montré qu'il y avait une différence statistiquement significative au niveau de tous les volumes faciaux étudiés: les cavités orbitaires $(p=0,022<0,05)$, la cavité ethmoïdo-nasale $(p=0,000<0,05)$, la cavité buccale $(p=0,000<0,05)$, les sinus maxillaires $(p=0,000<0,05)$, ainsi que pour le volume global de toutes les cavités $(p=0,000<0,05)$ (Tab. 1).

En moyenne, les hommes ont des volumes plus grands que les femmes de 17,38 \% au niveau des cavités orbitaires, $24 \%$ pour la cavité ethmoïdo-nasale, $28,5 \%$ pour la cavité buccale, $27 \%$ pour les sinus maxillaires et $26 \%$ au niveau de la totalité des cavités étudiées.

De même, les rapports du volume de chacune des cavités sur le volume total ont été trouvés, ainsi que leurs moyennes et écarts-types, et les comparaisons faites. Aucune différence significative n'a été trouvée au niveau du rapport de chaque cavité sur le volume total (Tab. 2).

\section{Discussion}

Les résultats ont montré l'existence d'un dimorphisme sexuel au niveau des cavités orbitaires, ethmoïdo-nasales, buccales et des sinus maxillaires. Ceci est en adéquation avec les études en 2D [17,20] 
Tableau 2

Statistiques descriptives et comparaison des rapports volumétriques entre les deux groupes hommes/femmes.

\begin{tabular}{|l|c|c|c|c|c|c|c|}
\cline { 2 - 7 } \multicolumn{1}{c|}{} & Groupe & $N$ & Minimum & Maximum & Moyenne & Ecart-type & $p$ \\
\hline \multirow{2}{*}{$\begin{array}{l}\text { V.cavités } \\
\text { orbitaires/V.total }\end{array}$} & Homme & 30 & 0,047 & 0,136 & 0,10213 & 0,022575 & 0,115 \\
\cline { 2 - 8 } & Femme & 30 & 0,053 & 0,232 & 0,11398 & 0,033704 & \\
\hline \multirow{2}{*}{$\begin{array}{l}\text { V.cavité } \\
\text { ethmö̈do-nasale/V.total }\end{array}$} & Homme & 30 & 0,155 & 0,281 & 0,19516 & 0,028947 & 0,645 \\
\cline { 2 - 7 } & Femme & 30 & 0,136 & 0,251 & 0,19819 & 0,021147 & \\
\hline \multirow{2}{*}{$\begin{array}{l}\text { V.cavité } \\
\text { buccale/V.total }\end{array}$} & Homme & 30 & 0,298 & 0,571 & 0,49298 & 0,053400 & 0,442 \\
\cline { 2 - 7 } & Femme & 30 & 0,406 & 0,558 & 0,48383 & 0,036505 & \\
\hline $\begin{array}{l}\text { V.sinus } \\
\text { maxillaires/V.total }\end{array}$ & Homme & 30 & 0,079 & 0,310 & 0,20973 & 0,042441 & 0,866 \\
\cline { 2 - 7 } & Femme & 30 & 0,126 & 0,267 & 0,20797 & 0,037659 & \\
\hline
\end{tabular}

comparant la base du crâne, dans le sens antéropostérieur, la hauteur faciale [6], ou bien encore la morphologie faciale entre les deux sexes: les mesures linéaires de la face sont plus grandes chez les hommes $[1,6,27,33]$ et le complexe cranio-facial est, en moyenne, de $5 \%$ à $9 \%$ plus grand chez les hommes que chez les femmes [32]. Plusieurs auteurs se sont intéressés à la forme plus qu'à la taille, Wei [33], dans son étude sur une population chinoise, a trouvé que toutes les mesures linéaires étaient supérieures chez les hommes alors que les mesures angulaires étaient identiques d'où une similitude de forme au niveau de la face entre les deux sexes. Halazonetis [16] a trouvé une légère différence de forme au niveau du profil, mais cela peut être dû à l'échantillon qui comprenait des sujets ayant toujours un potentiel de croissance. Starck et Epker [30], en étudiant les rapports relatifs et non les mesures céphalométriques absolues entre deux groupes de jeunes adultes de sexe différent, n'ont pas trouvé de différence de forme au niveau de la face, ce qui est proche des résultats de notre étude, si nous extrapolons au niveau volumétrique en passant du 2D au 3D, concernant les rapports des volumes des cavités étudiées sur le volume total. Le fait que nous n'ayons pas trouvé de différences significatives relatives entre les deux groupes malgré une différence volumétrique absolue des cavités, prouverait que la forme pourrait être similaire malgré une différence de taille.

Du point de vue volumétrique, les études se sont surtout intéressées aux tissus mous, ce qui est probablement dû à la possibilité de réalisation et de mesure avec des moyens non-invasifs surtout lorsque l'étude est faite sur un large échantillon. Sforza, et al. [26], en étudiant la région orbitaire en 3D, prenant des points de repère au niveau des tissus mous, ont conclu que toutes les dimensions étaient plus importantes chez les hommes, à l'exception de la hauteur orbitaire qui est identique chez les hommes et les femmes, ce qui est en accord avec nos résultats au niveau orbitaire, bien que les deux études ne soient pas comparables par leur méthodologie. Ferrario, et al. [11-14] ont utilisé la «photométrie infrarouge » pour étudier les volumes faciaux, toujours au niveau des tissus mous; le dimorphisme sexuel se manifeste au niveau de la face avec une prépondérance au niveau du tiers inférieur, les hommes ayant des faces plus grandes dans les trois dimensions et les volumes masculins absolus ont des valeurs supérieures de 6 à $7 \%$ alors qu'il n'y aurait pas de différence quant à la forme faciale. Nous avons trouvé des pourcentages supérieurs au niveau des volumes absolus des cavités étudiées, ceci pourrait être dû au fait que nos mesures sont prises sur des images scanners plus représentatives donc non comparables aux points de repère localisés sur les tissus mous. Si les différences des mesures linéaires unidimensionnelles entre les deux sexes sont de $5 \%$ à $9 \%$ [32], on pourrait s'attendre, au niveau volumétrique tridimensionnel, à des différences de $17 \%$ à $28,5 \%$, comme nous l'avons trouvé dans notre étude.

\section{Conclusion}

Limagerie 3D est un outil précis nous permettant d'étudier la morphologie cranio-faciale dans ses trois dimensions, ce qui était jusque-là impossible avec les radiographies céphalométriques.

Le dimorphisme sexuel au niveau des mesures linéaires cranio-faciales avait été largement démontré, cette étude a permis de comparer des valeurs volumétriques cranio-faciales chez de jeunes adultes de sexe différent, en utilisant des acquisitions scanner 3D. 
Les moyennes des volumes des cavités étudiées: orbitaires, ethmoïdo-nasales, buccales et des sinus maxillaires sont plus grandes chez les hommes. Mais le dimorphisme sexuel n'a pas été mis en évidence au niveau des rapports des volumes de chacune des cavités étudiées sur le volume facial total, il y aurait donc une différence cranio-faciale de taille, et non de forme, entre les hommes et les femmes.

\section{Bibliographie}

[1] Buschang PH, Nass GG, Walker GF. Principal components of craniofacial growth for white Philadelphia males and females between 6 and 22 years of age. Am J Orthod Dentofacial Orthop 1982;82:508-512.

[2] Cho HJ, Redmond WR. A three-dimensional cephalometric analysis. J Clin Orthod 2009;63:235-252.

[3] Dah-Jouonzo H, Baron P, Faure J, Treil J, Borianne Ph. Mesures volumétriques des bases osseuses maxillaires et des cavités aériennes de la face en imagerie 3D. Int Orthod 2005;3:269-289

[4] Dah-Jouonzo H, Baron P, Faure J. Liaisons entre volumes des cavités aériennes et des bases osseuses maxillaires et paramètres de céphalométrie 3D. Orthod Fr 2007:78:265-281

[5] Dean D, Hans MG, Bookstein FL, Subramanvan K. Threedimensional Bolton-Brush Growth Study landmark data: ontogeny and sexual dimorphism of the Bolton standards cohort. Cleft Palate Craniofac J 2000;37:145-156.

[6] De Freitas LMA, Pinzan A, Janson G, Freitas KMS, De Freitas MR. Facial height comparison in young white and black Brazilian subjects with normal occlusion. Am J Orthod Dentofacial Orthop 2007;131:706.e1-706.e6.

[7] Faure J, Baron P, Treil J. Analyse céphalométrique tridimensionnelle: diagnostic des dysmorphies antéropostérieures et verticales. Orthod Fr 2005;76:91-110.

[8] Faure J, Marshal-Sixou C, Dah-Jouonzo H, Oueiss A, Baron P. Analyse céphalométrique tridimensionnelle à 14 points : compensations des dysmorphies maxillo-faciales. Orthod Fr 2008;79:251-261.

[9] Faure J, Oueiss A, Marchal-Sixou C, Braga J, Treil J. Céphalométrie tridimensionnelle : applications en clinique et en recherche. Orthod Fr 2008;79:13-30.

[10] Faure J, Treil J, Borianne P, Casteigt J, Baron P. Céphalométrie tridimensionnelle en orthopédie dentofaciale. Les possibilités actuelles du logiciel Cepha3DT. Orthod Fr 2002;73:19-37.

[11] Ferrario VF, Sforza C, Poggio CE, Serrao G, Miani A Jr. A three-dimensional study of sexual dimorphism in the human face. Int J Adult Orthod Orthognath Surg 1994:9:303-310

[12] Ferrario VF, Sforza C, Serrao G, Miani A Jr. A computerized non-invasive method for the assessment of human facial volume. J Craniomaxillofac Surg 1995;23:280-286.

[13] Ferrario VF, Sforza C, Poggio CE, Schmitz JH. Soft-tissue facial morphometry from 6 years to adulthood: a threedimensional growth study using a new modeling. Plast Reconstr Surg 1999;103:768-778.
[14] Ferrario VF, Sforza C, Poggio CE, Schmitz JH. Facial volume changes during normal human growth and development. Anat Rec 1998;250:480-487.

[15] Forsberg CM. Facial morphology and ageing: a longitudinal investigation of young adults. Eur J Orthod 1979;1:15-23

[16] Halazonetis DJ. Morphometric evaluation of softtissue profile shape. Am J Orthod Dentofacial Orthop 2007;131:481-489.

[17] Henneberke M, Prahl-Andersen B. Cranial base growth for Dutch boys and girls: a multilevel approach. Am J Orthod Dentofacial Orthop 1994;106:503-512.

[18] Hennessy RJ, Moss JP. Facial growth: separating shape from size. Eur J Orthod 2001;23:275-285.

[19] Houston WJB. The analysis of errors in orthodontic measurements. Am J Orthod 1983;83:382-390.

[20] Ingerslev $\mathrm{CH}$, Solow B. Sex differences in craniofacial morphology. Acta Odontol Scand 1975;33:85-94.

[21] Kau CH, Richmond S. Three-dimensional analysis of facial morphology surface changes in untreated children from 12 to 14 years of age. Am J Orthod 2008;134:751-760.

[22] Kau CH, Zhurov A, Richmond S, Cronin A, Savio C, Mallorie C. Facial templates: a new perspective in three dimensions. Orthod Craniofac Res 2006;9:10-17.

[23] Kau CH, Zhurov A, Scheer R, Bouwman S, Richmond $\mathrm{S}$. The feasibility of measuring three-dimensional facial morphology in children. Orthod Craniofac Res 2004;7:198-204

[24] Nabbout F, Faure J, Baron P, Braga J, Treil J. Centres de résistance des groupes dentaires et mécanique orthodontique. Int Orthod 2007;5:437-446.

[25] Sampeur M, Boileau MJ. Caractéristiques des muscles masticateurs en tomodensitométrie : variations en fonction de la typologie squelettique. Orthod Fr 2008;79:151-159.

[26] Sforza C, Grandi G, Catti F, Tommasi DG, Ugolini A, Ferrario VF. Age- and sex-related changes in the soft tissues of the orbital region. Forensic Sci Int 2009; 185(1-3):115.e1-8.

[27] Siriwat PP, Jarabak JR. Malocclusion and facial morphology is there a relationship? An epidemiologic study. Angle Orthod 1985;55(2):127-138.

[28] Sobotta. Atlas d'Anatomie Humaine. Tome I. Tête, Cou, Membres Supérieurs. $3^{e}$ éd. fr. Editions Médicales Internationales 1994:30-32.

[29] Sosa FA, Graber TM, Muller TP. Postpharyngeal lymphoid tissue in Angle Class I and Class II malocclusions. Am J Orthod 1982;81(4):299-309.

[30] Starck WJ, Epker BN. Cephalometric analysis of profile nasal esthetics. Part I. Method and normative data. Int J Adult Orthod Orthognath Surg 1996;11(2):91-103.

[31] Treil J, Casteigt J, Faure J, Horn A, Labadie MP. Orthodontie et tomodensitométrie. Rev Orthop Dento Faciale 2003;37:9-21.

[32] Ursi WJS, Trotman CA, McNamara JA Jr, Behrents RG. Sexual dimorphism in normal craniofacial growth. Angle Orthod 1993;63:47-56.

[33] Wei SHY. Craniofacial variations, sex differences and the nature of prognathism in Chinese subjects. Angle Orthod 1969;39:303-315. 